\title{
Dynamics of information in the presence of deformation
}

\author{
N. Metwally \\ Math. Dept., Faculty of Science, South Valley University, Aswan, Egypt. \\ E.mail: Nmetwally@gmail.com
}

\begin{abstract}
The entanglement of atomic system consists of two atoms interacts with a deformed cavity mode is quantified by the means of Bloch vectors and the cross dyadic of the traveling state inside the cavity. For large value of the deformation the amplitude of Bloch vectors decrease very fast and consequently the traveling state turns into mixed state quickly. The generated entangled state is used as quantum channel to implement quantum teleportation protocol. It is shown that both of the deformed parameter and the number of photons inside the cavity play a central role on controlling the fidelity of the transmitted information.
\end{abstract}

\section{Introduction}

Controlling quantum coherence is one of the most fundamental issues in quantum information processing [1]. From a practical point of view the investigating of the behavior of entanglement in the presences of noise is very important, where there are many studies that has been done in different directions. Among these noise is the deformation, which arise from the defects of devices [2, 3, 4. So, in the presence of deformation, one can generate a deformed entangled state and consequently there will be a deformation on the information processing.

The quantization of the field in the presence of deformed operators has been investigated in different topics, where the relation between the deformed and non deformed operators have been obtained in [5, 6, 7]. As an applications of the deformed operators, Mancini and Manko have compared the dynamics of information which is coded in deformed cat state with non-deformed cat state [8]. Also, in [9] the holonomic quantum gates using isospectral deformations of an Ising model have been constructed. The survival of quantum coherence against dissipation provided to superpose distinguishable coherent states of suitable deformed field has been investigated in [10]. Recently, Metwally and et.al, have showed that the nonclassical properties of a two qubits state can be enhanced via deformed operators[11].

These efforts motivate us to introduce a different application of the deformation. In this work, we consider a system consists of of two separable atoms interacts with a cavity mode with multiphotons. The operators which describe the field are assumed to be deformed. We investigate the behavior of entanglement which is generated between the two atoms. Also, the dynamics of the Bloch vectors are investigated for this deformed atomic system. Finally, we use the generated entangled state between the two atoms as quantum channel to implement the quantum teleportation protocol. The effect of the deformation on the fidelity of the transmitted information is investigated.

This paper is organized as follows: In Sec.2, we introduce the model and its solution by means of the Bloch vectors and the cross dyadic. The entanglement and the dynamics of the Bloch vectors are studied in Sec.3. The quantum teleportation protocol is the subject of Sec.4. Finally, we summarize our results in Sec.5, 


\section{$2 \quad$ Model}

Let us assume that we have atomic system consists of two atoms interacts with cavity mode with $m$ photons. The annihilation and creation operators which describe the cavity mode are assumed to be deformed. The deformed operators result as a distortion of the usual annihiliation and creation operators [2, 6]. In the rotating wave approximation, the interaction of the cavity mode and the atomic systems is described by the Hamilation ,

$$
\hat{H}=\varpi_{0} a_{q}^{\dagger} a_{q}+\frac{1}{2} \omega\left(\sigma_{z}+\tau_{z}\right)+\lambda_{1}\left(\sigma_{+} a_{q}^{m}+\sigma a_{q}^{\dagger m}\right)+\lambda_{2}\left(\tau_{+} a_{q}^{m}+\tau_{-} a_{q}^{\dagger m}\right),
$$

where, $\varpi_{0}$ is the frequencies of the field, $\omega_{i}$ are the atomic transition frequency, $\lambda_{i}, i=1,2$ are the coupling constants between the atoms and the field, $a_{q}$ and $a_{q}^{\dagger}$ are deformed annihilation and creation operators, which can be described by means of the non-deformed operators $a$ and $a^{\dagger}$ as,

$$
a_{q}=a f(n), \quad a_{q}^{\dagger}=a^{\dagger} f(n),
$$

where $f(\hat{n})$ is a function of the number of photons $\bar{n}=a^{\dagger} a$. The deformed operators $a_{q}$ and $a_{q}^{\dagger}$ satisfy the commutation relations,

$$
\begin{aligned}
{\left[a_{q}, a_{q}^{\dagger}\right] } & =(\hat{n}+1) f^{2}(\hat{n}+1)-\hat{n} f^{2}(\hat{n}) \\
{\left[a_{q}, n\right] } & =a_{q}, \quad\left[a_{q}^{\dagger}, n\right]=-a_{q}^{\dagger} .
\end{aligned}
$$

The operators $\sigma_{ \pm}=\sigma_{x} \pm i \sigma_{y}, \tau_{ \pm}=\tau_{x} \pm i \tau_{y}$ and $\sigma_{z}, \tau_{z}$ are the raising (lowering) and inversion operators for the two atoms. The operators $\sigma_{i}$ and $\tau_{i}, i=x, y, z$ are the Pauli's operators for the first and the second atoms respectively. In this treatment, the function $f(\hat{n})$ represents what is called the $q$-deformation and it is defined as

$$
f(\hat{n})=\sqrt{\frac{1-q^{n}}{n(1-q)}} .
$$

In the invariant sub-space of the global system, we can assume a set of complete basis of the field-atomic system as $|e, e, n\rangle,|e, g, n+2\rangle,|g, e, n+2\rangle$ and $|g, g, n+2\rangle$. Now, let the initial state of the atomic system, $\left|\psi_{a}(0)\right\rangle=a_{1}|e e\rangle+a_{2}|e g\rangle+a_{3}|g e\rangle+a_{4}|g g\rangle$, while the field is initially prepared in the coherent state $\left|\psi_{f}(0)\right\rangle=\sum_{n=0}^{\infty} W_{n}|n\rangle, W_{n}=\frac{\bar{n}^{n}}{\sqrt{n !}} \operatorname{Exp}\left(-\frac{1}{2}|\bar{n}|^{2}\right)$.

For a convenient notations the density operator of the atomic system is described by the Bloch vectors and the cross dyadic as [12, 13],

$$
\rho_{a}(0)=\frac{1}{4}\left(+\vec{s}_{0} \cdot \sigma^{\downarrow}+\vec{t}_{0} \cdot \tau^{\downarrow}+\vec{\sigma} \cdot{ }^{\downarrow} \vec{C}(0) \cdot \tau^{\downarrow}\right)
$$

where,

$$
\vec{s}_{0}=\left(s_{x}(0), s_{y}(0), s_{z}(0)\right), \quad \vec{t}_{0}=\left(t_{x}(0), t_{y}(0), t_{z}(0)\right),
$$

are the initial Bloch vectors of the two atoms respectively, with

$$
\begin{aligned}
& s_{x}(0)=\left(a_{1} a_{3}^{*}+a_{3} a_{1}^{*}\right)+\left(a_{2} a_{4}^{*}+a_{4} a_{2}^{*}\right), s_{y}(0)=i\left(a_{3} a_{1}^{*}-a_{1} a_{3}^{*}\right)+i\left(a_{4} a_{2}^{*}-i a_{2} a_{4}^{*}\right), \\
& s_{z}(0)=\left|a_{1}\right|^{2}+\left|a_{2}\right|^{2}-\left|a_{3}\right|^{2}-\left|a_{4}\right|^{2}, t_{x}(0)=\left(a_{1} a_{2}^{*}+a_{2} a_{1}^{*}\right)+\left(a_{3} a_{4}^{*}+a_{4} a_{3}^{*}\right), \\
& t_{y}(0)=i\left(a_{2} a_{1}^{*}-a_{1} a_{2}^{*}\right)+i\left(a_{4} a_{3}^{*}-a_{3} a_{4}^{*}\right), t_{z}(0)=\left|a_{1}\right|^{2}-\left.a_{2}\right|^{2}+\left|a_{3}\right|^{2}-\left|a_{4}\right|^{2} .
\end{aligned}
$$


and the elements of the initial cross dyadic between the two atoms are given by,

$$
\begin{aligned}
& c_{x x}(0)=a_{1} a_{4}^{*}+a_{2} a_{3}^{*}+a_{3} a_{2}^{*}+a_{4} a_{1}^{*}, c_{x y}(0)=i\left(a_{4} a_{1}^{*}-a_{1} a_{4}^{*}\right)+i\left(a_{2} a_{3}^{*}-a_{3} a_{2}^{*}\right), \\
& c_{x z}(0)=\left(a_{1} a_{3}^{*}+a_{3} a_{1}^{*}\right)-\left(a_{2} a_{4}^{*}+a_{4} a_{2}^{*}\right), c_{y x}(0)=i\left(a_{4} a_{1}^{*}-a_{1} a_{4}^{*}\right)+i\left(a_{3} a_{2}^{*}-a_{2} a_{3}^{*}\right), \\
& c_{y y}(0)=\left(a_{3} a_{2}^{*}+a_{2} a_{3}^{*}\right)-\left(a_{4} a_{1}^{*}+a_{1} a_{4}^{*}\right), c_{y z}(0)=i\left(a_{2} a_{4}^{*}-a_{4} a_{2}^{*}\right)+i\left(a_{3} a_{1}^{*}-a_{1} a_{3}^{*}\right), \\
& c_{z x}(0)=\left(a_{1} a_{2}^{*}+a_{2} a_{1}^{*}\right)-\left(a_{3} a_{4}^{*}+a_{4} a_{3}^{*}\right), c_{z y}(0)=i\left(a_{2} a_{1}^{*}-a_{1} a_{2}^{*}\right)+i\left(a_{3} a_{4}^{*}-a_{4} a_{3}^{*}\right), \\
& c_{z z}(0)=\left|a_{1}\right|^{2}-\left|a_{2}\right|^{2}-\left|a_{3}\right|^{2}+\left|a_{4}\right|^{2} .
\end{aligned}
$$

Also, the density operator of the field is given by,

$$
\rho_{f}(0)=\sum_{n=0}^{\infty} W_{n}^{2}|n\rangle\langle n| .
$$

Then using the Hamiltonian (1), the initial state of the atomic system (5), and the initial state of the field (9) one gets the time evolution of the density operator of the field and the atomic system, where $\lambda_{1}=\lambda_{2}=\lambda, \omega_{1}=\omega_{2}=\omega$, and $\omega_{0}-\omega=\Delta=0$. After tracing out the state of the field, the density operator of the atomic system is given by

$$
\rho_{a}(t)=\frac{1}{4}\left(1+\vec{s} \cdot \sigma^{\downarrow}+\vec{t} \cdot \tau^{\downarrow}+\vec{\sigma} \cdot \overleftrightarrow{\complement} \operatorname{rrow} C \cdot \tau^{\downarrow}\right)
$$

where,

$$
\begin{aligned}
& s_{x}(t)=\sum_{n=0}^{\infty}\left(c_{n}^{(1)} c_{n-2}^{*(3)}+c_{n}^{(2)} c_{n-2}^{*(4)}+c_{n}^{(3)} c_{n+2}^{*(1)}+c_{n}^{(4)} c_{n+2}^{*(2)}\right), \\
& s_{y}(t)=\sum_{n=0}^{\infty}\left(-i c_{n}^{(1)} c_{n-2}^{*(3)}-i c_{n}^{(2)} c_{n-2}^{*(4)}+i c_{n}^{(3)} c_{n+2}^{*(1)}+i c_{n}^{(4)} c_{n+2}^{*(2)}\right), \\
& s_{z}(t)=\sum_{n=0}^{\infty}\left(\left|c_{n}^{(1)}\right|^{2}+\left|c_{n}^{(2)}\right|^{2}-\left|c_{n}^{(3)}\right|^{2}-\left|c_{n}^{(4)}\right|^{2}\right), \\
& t_{x}(t)=\sum_{n=0}^{\infty}\left(c_{n}^{(1)} c_{n-2}^{*(2)}+c_{n}^{(2)} c_{n+2}^{*(1)}+c_{n}^{(3)} c_{n-2}^{*(4)}+c_{n}^{(4)} c_{n+2}^{*(3)}\right), \\
& t_{y}(t)=\sum_{n=0}^{\infty}\left(-i c_{n}^{(1)} c_{n-2}^{*(2)}+i c_{n}^{(2)} c_{n+2}^{*(1)}-i c_{n}^{(3)} c_{n-2}^{*(4)}+i c_{n}^{(4)} c_{n+2}^{*(3)}\right), \\
& t_{z}(t)=\sum_{n=0}^{\infty}\left(\left|c_{n}^{(1)}\right|^{2}-\left|c_{n}^{(2)}\right|^{2}+\left|c_{n}^{(3)}\right|^{2}-\left|c_{n}^{(4)}\right|^{2}\right),
\end{aligned}
$$


and the elements of the cross dyadic are,

$$
\begin{aligned}
c_{x x}(t)= & \sum_{n=0}^{\infty}\left(c_{n}^{(1)} c_{n-4}^{*(4)}+c_{n}^{(4)} c_{n+4}^{*(1)}+c_{n}^{(2)} c_{n}^{*(3)}+c_{n}^{(3)} c_{n}^{*(2)}\right), \\
c_{x y}(t)= & i \sum_{n=0}^{\infty}\left(-c_{n}^{(1)} c_{n-4}^{*(4)}+c_{n}^{(4)} c_{n+4}^{*(1)}+c_{n}^{(2)} c_{n}^{*(3)}-c_{n}^{(3)} c_{n}^{*(2)}\right), \\
c_{x z}(t)= & \sum_{n=0}^{\infty}\left(c_{n}^{(1)} c_{n-2}^{*(3)}+c_{n}^{(3)} c_{n+2}^{*(1)}-c_{n}^{(2)} c_{n-2}^{*(4)}+c_{n}^{(4)} c_{n+2}^{*(2)}\right), \\
c_{y x}(t)= & i \sum_{n=0}^{\infty}\left(-c_{n}^{(1)} c_{n-4}^{*(4)}+c_{n}^{(4)} c_{n+4}^{*(1)}-c_{n}^{(2)} c_{n}^{*(3)}+c_{n}^{(3)} c_{n}^{*(2)}\right) \\
c_{y y}(t)= & -\sum_{n=0}^{\infty}\left(c_{n}^{(1)} c_{n-4}^{*(4)}+c_{n}^{(4)} c_{n+4}^{*(1)}-c_{n}^{(2)} c_{n}^{*(3)}-c_{n}^{(3)} c_{n}^{*(2)}\right), \\
c_{y z}(t)= & i \sum_{n=0}^{\infty}\left(-c_{n}^{(1)} c_{n-2}^{*(3)}+c_{n}^{(3)} c_{n+2}^{*(1)}+c_{n}^{(2)} c_{n-2}^{*(4)}+c_{n}^{(4)} c_{n+2}^{*(2)}\right), \\
c_{z x}(t)= & \sum_{n=0}^{\infty}\left(c_{n}^{(1)} c_{n-2}^{*(2)}+c_{n}^{(2)} c_{n+2}^{*(1)}-c_{n}^{(3)} c_{n-2}^{*(4)}-c_{n}^{(4)} c_{n+2}^{*(3)}\right), \\
c_{z y}(t)= & i \sum_{n=0}^{\infty}\left(-c_{n}^{(1)} c_{n-2}^{*(2)}+c_{n}^{(2)} c_{n+2}^{*(1)}+c_{n}^{(3)} c_{n-2}^{*(4)}-c_{n}^{(4)} c_{n+2}^{*(3)}\right), \\
c_{z z}(t)= & \sum_{n=0}^{\infty}\left(\left|c_{n}^{(1)}\right|^{2}-\left|c_{n}^{(2)}\right|^{2}-\left|c_{n}^{(3)}\right|^{2}+\left|c_{n}^{(4)}\right|^{2}\right) \\
&
\end{aligned}
$$

with,

$$
\begin{aligned}
& c_{n}^{(1)}(t)=a_{1} W_{n}-\nu_{1}\left(a_{1} \nu_{1} W_{n}+a_{4} \nu_{2} W_{n+2}\right) \frac{\sin ^{2} \mu_{n} t}{\mu_{n}^{2}}-i \nu_{1}\left(a_{2}+a_{3}\right) W_{n+1} \frac{\sin 2 \mu_{n} t}{2 \mu_{n} t} \\
& c_{n}^{(2)}(t)=W_{n+1}\left(a_{2} \cos ^{2} \mu_{n} t-a_{3} \sin ^{2} \mu_{n} t\right)-i\left(a_{1} \nu_{1} W_{n}+a_{4} \nu_{2} W_{n+2}\right) \frac{\sin 2 \mu_{n} t}{2 \mu_{n} t} \\
& c_{n}^{(3)}(t)=W_{n+1}\left(a_{3} \cos ^{2} \mu_{n} t-a_{2} \sin ^{2} \mu_{n} t\right)-i\left(a_{1} \nu_{1} W_{n}+a_{4} \nu_{2} W_{n+2}\right) \frac{\sin 2 \mu_{n} t}{2 \mu_{n} t} \\
& c_{n}^{(4)}(t)=a_{4} W_{n+2}-\nu_{2}\left(a_{1} \nu_{1} W_{n}+a_{4} \nu_{2} W_{n+2}\right) \frac{\sin ^{2} \mu_{n} t}{\mu_{n}^{2}}-i \nu_{2}\left(a_{2}+a_{3}\right) W_{n+1} \frac{\sin 2 \mu_{n} t}{2 \mu_{n} t}
\end{aligned}
$$

where, $\mu_{n}=\frac{1}{\sqrt{2}} \sqrt{\nu_{1}^{2}(n)+\nu_{2}^{2}(n)}, \nu_{1}(n)=\lambda \sqrt{(n+m) ! / n !}$ and $\nu_{2}(n)=\lambda \sqrt{(n+2 m) ! /(n+m) !}$.

\section{Dynamics of Entanglement}

Since we use a measure of entanglement depends on the Bloch vectors and the cross dyadic of the two atoms, it is important to shed light on the dynamics of these vectors. This study gives us a perception in the form of the state of the two atoms as it passes through the deformed cavity. We assume that the two atoms are identical, so the dynamics of $|\vec{s}|$ for the first atom and $|\vec{t}|$ for the second atom has the same behavior.

Fig.(1) shows the dynamics of the Bloch vectors for different values of the deformation. For free deformation cavity, the amplitude of the Bloch vectors decreases as $\lambda t$ increases. 

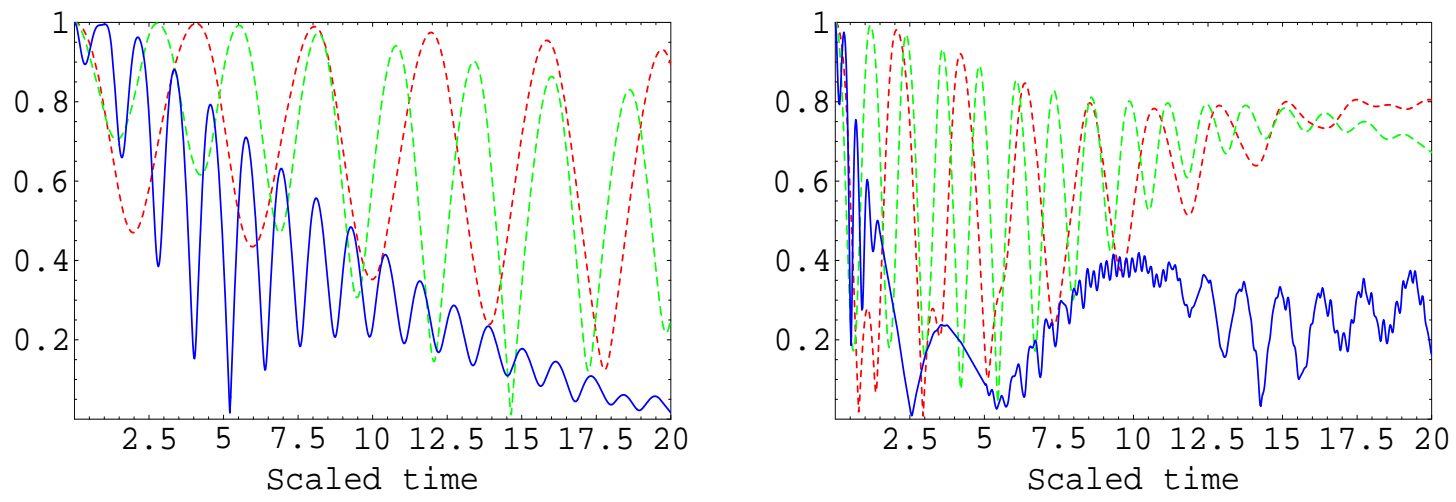

Figure 1: The Bloch vector for the travailing state (17), where the deformed parameter $q=0,0.5,0.9$ and the mean photon number, $\bar{n}=10$ for the dot, dash -dot and solid curves,respectively (a) $m=1$ and (b) $m=2$.

Due to the flocculating behavior, the travelling state through the cavity turns into mixed and pure state several time. As one consider the deformation, the amplitude of the Bloch vectors decrease more and the minimum values are always smaller than that depicted for the free deformation case (dot-curves). However as one increases the deformed parameter $(q=0.9)$, the amplitude of the Bloch vectors decrease faster as shown in Fig. $(1 a)$. This means that the state of the two qubits turns into a mixed state faster for larger values of the deformity.

In Fig.(1b), the number of photons inside the cavity is increased $(m=2)$. It is clear that for free deformation the amplitude of the Bloch vectors oscillates so fast and increases as time increases. So, by increasing the number of photons inside the cavity, one can increase the purity of the travelling state. In the presence of deformation $(q=0.5)$, the behavior of the amplitude of the Bloch vectors is similar to that depicted for the free deformation case. However as one increases the deformity parameter more $(q=0.9)$, the amplitude of the Bloch vectors increases. From Fig.(1a) and Fig(1b), it is clearly that by increasing the number of photons inside the cavity, one can avoid the defected which results from the deformed cavity.

On the other hand, when the Bloch vectors vanish and the entanglement still survival between the two atoms, this means that an entangled state of Werner type [16, 13] has been generated and it can be written as:

$$
\rho_{w}=\frac{1}{4}\left(1+x_{1} \sigma_{x} \tau_{x}+x_{2} \sigma_{y} \tau_{y}+x_{3} \sigma_{z} \tau_{z}\right)
$$

However, at certain time say $(\mathrm{t}=2.5,5)$ as shown in Fig.(1a), the amplitudes of the Bloch vectors $|\vec{s}|=|\vec{t}|=1$ and the entanglement vanishes (see Fig.(2a)), this means that the atomic system turns into itsd initial state.

To quantify the degree of entanglement between the two atoms, we use a measure defined by means of the Bloch vectors and the cross dyadic. The entangled dyadic is defined as

$$
{ }^{\downarrow} \vec{E}={ }^{\downarrow} \vec{C}-\vec{s} t^{\downarrow}
$$

where ${ }^{\downarrow} \vec{C}$ is a $3 \times 3$ matrix which is defined by(12) and $\vec{s} t^{\downarrow}$ is also a $3 \times 3$ matrix whose elements can be obtained from (11). The degree of entanglement is defined as in [14, 15]

$$
\mathcal{E}=\operatorname{tr}\left\{{ }^{\downarrow} @ \text { rrow } E^{\top} \cdot \vec{\triangleright} \text { rrow } E\right\},
$$



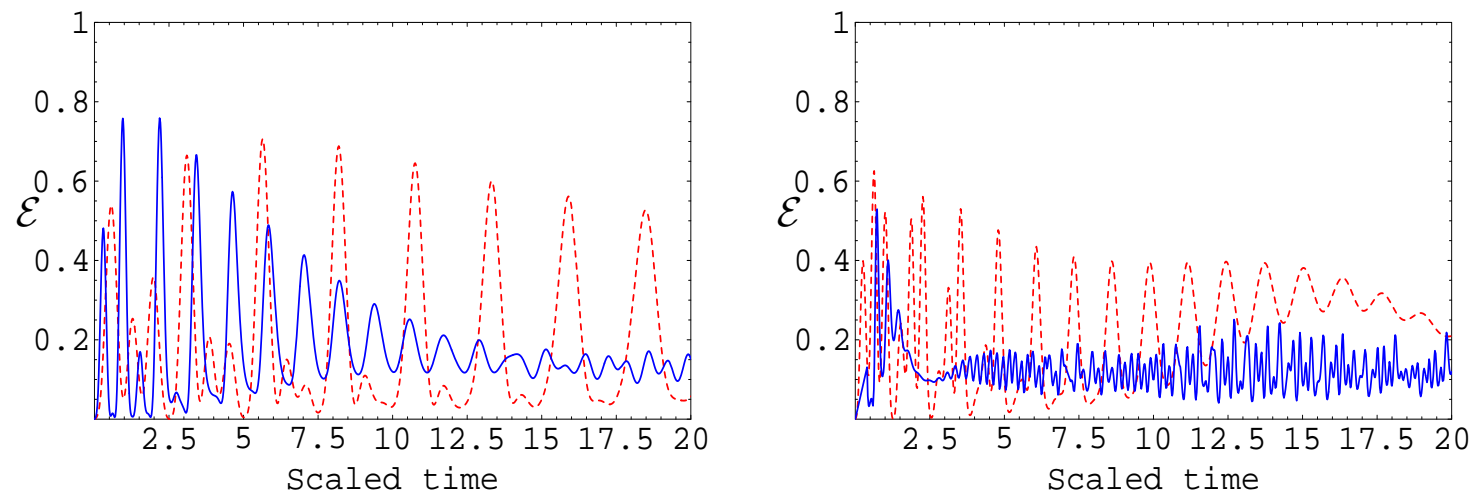

Figure 2: The degree of entanglement $\mathcal{E}$ generated between the two atoms prepared initially in the state (17) The deformed parameter $q=0.5,0.9$ and the mean photon numbers, $\bar{n}=10$ for the dot and solid curves respectively (a) $m=1$ and (b) $m=2$.

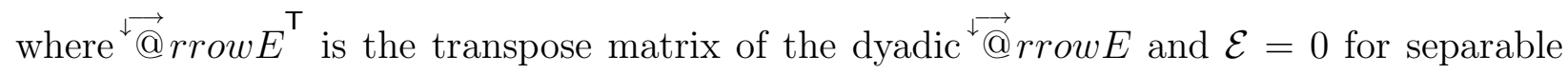
states.

Fig (2), describes the dynamics of entanglement which is generated between two atoms prepared initially in a separable state define by,

$$
\rho_{a}=\frac{1}{4}\left(1+s_{z}+t_{z}+c_{z z}\right)
$$

Fig.(2a), shows the behavior of the degree of entanglement for $m=1$. Since we start with a separable atomic system, the degree of entanglement, $\mathcal{E}=0$ at $\lambda t=0$. However, for $\lambda t>0$, an entangled state generated between the two atoms. For $q=0.5$, one can easily see that the entanglement of the two atoms decreases as time increases( dot-curves). However, $\mathcal{E}$ goes to zero for a very small finite time and suddenly increases. As one increases the deformity parameter $(q=0.9)$, the degree of entanglement decreases gradually but does not vanish. It is clear that, the entanglement tends to zero several time for small values of the deformity parameter. On the other hand, for larger values of the deformity, $\mathcal{E}$ goes to zero only limited time. The dynamics of the entanglement for $m=2$ is displayed in Fig.(2b), where it is smaller than that depicted in Fig.(1a). However, the entanglement oscillates so fast as one increases the value of the deformed parameter. It is clear that, the possibility of obtaining a long lived entanglement is increases as one increases the value of the deformed parameter, where we can see that the entanglement is zero only when $\lambda t=0$.

One can conclude that the number of photons plays as a control parameter, where one can improve the degree of entanglement between the two atoms, by increasing the number of photons inside the cavity. Also, the long-lived entanglement between the two atoms can be obtained by increasing the deformation.

\section{Teleportation}

In this section, the generated entangled state (10) is employed to implement the original quantum teleportation protocol [17]. This protocol is used to send unknown quantum state between two users Alice and Bob, by using local operations and classical communication. Now assume that, Alice is given unknown state defined by,

$$
\rho_{u}=\frac{1}{2}\left(1+\vec{s}_{u} \cdot \sigma\right),
$$



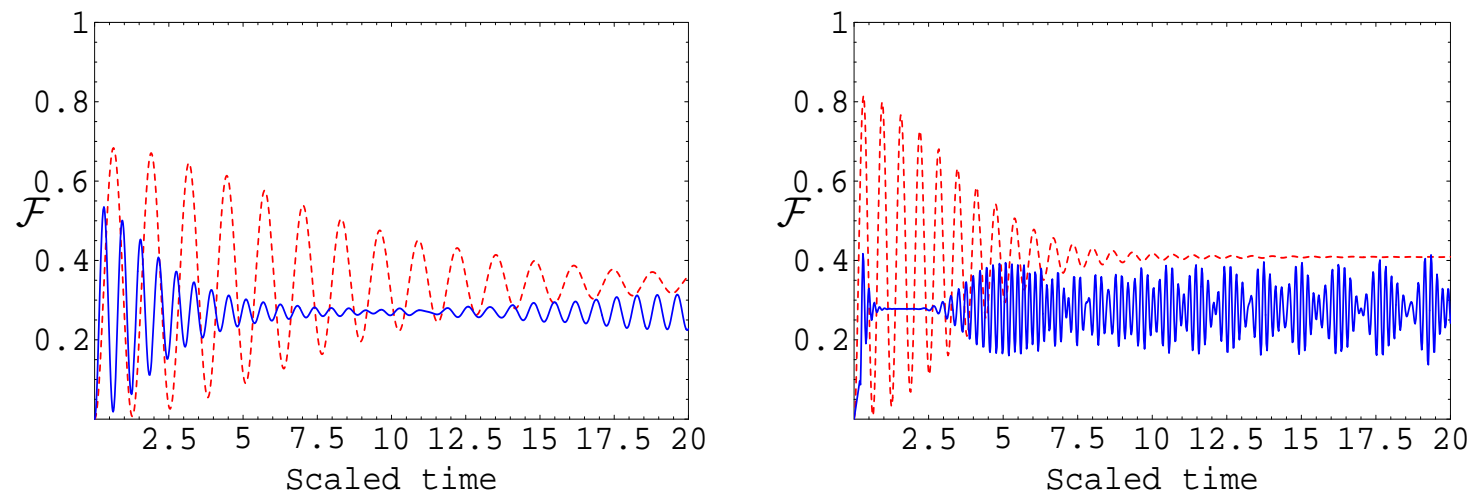

Figure 3: The Fidelity of the teleported state (18) with $s_{x_{u}}=1, s_{y_{u}}=s_{z_{u}}=0$, and the deformed parameter $q=0.5,0.9$ and $\bar{n}=10$ for the dot and solid curves respectively (a) $m=1$ and (b) $m=2$.

where the componnents of the Bloch vector $\vec{s}_{u}$ are given by

$$
s_{u_{x}}=\alpha \beta^{*}+\beta \alpha^{*}, s_{u_{y}}=i\left(\beta \alpha^{*}-\alpha \beta^{*}\right), s_{u_{z}}=|\alpha|^{2}-|\beta|^{2}, \text { where }|\alpha|^{2}+|\beta|^{2}=1 .
$$

To achieve the teleportation Protocol, the partners follow the following steps:

1. Alice performs the CNOT gate on her qubit and the given unknown qubit followed by Hadamard gate.

2. Alice measures her qubit and the unknown qubit randomly in one of the basis $|e e\rangle,|e g\rangle,|e e\rangle$ and $|g g\rangle$ and sends her results to Bob by using classical channel.

3. As soon as Bob receives the classical data from Alice, he applies a single qubit operation on his qubit depending on Alice's results. So, if Alice measures in the basis $|e e\rangle$, Bob will obtain the state.

$$
\rho_{B o b}=\frac{1}{2}\left(1+\vec{s}_{b} \cdot \vec{\sigma}\right)
$$

where, $s_{x_{b}}, s_{y_{b}}, s_{z_{b}}$ are the components of $\vec{s}_{b}$,

$$
\begin{aligned}
s_{x_{b}}= & |\alpha|^{2}\left(c_{n}^{(3)} c_{n-1}^{(* 4)}+c_{n}^{(4)} c_{n+2}^{(* 3)}\right)+\alpha \beta^{*}\left(c_{n}^{(4)} c_{n-1}^{(* 1)}+c_{n}^{(3)} c_{n}^{(* 2)}\right)- \\
& \alpha^{*} \beta\left(c_{n}^{(1)} c_{n-2}^{(* 2)}+c_{n}^{(3)} c_{n}^{(* 2)}\right)+|\beta|^{2}\left(c_{n}^{(1)} c_{n-2}^{(* 2)}+c_{n}^{(2)} c_{n+2}^{(* 1)},\right. \\
s_{y_{b}}= & |\alpha|^{2}\left(c_{n}^{(4)} c_{n+2}^{(* 3)}-c_{n}^{(3)} c_{n-2}^{(* 4)}\right)+\alpha \beta^{*}\left(c_{n}^{(4)} c_{n-1}^{(* 1)}-c_{n}^{(3)} c_{n}^{(* 2)}\right) \\
& -\alpha^{*} \beta\left(c_{n}^{(3)} c_{n}^{(* 2)}-c_{n}^{(1)} c_{n-2}^{(* 2)}\right)+|\beta|^{2}\left(c_{n}^{(2)} c_{n+2}^{(* 1)}-c_{n}^{(1)} c_{n-2}^{(* 2)}\right), \\
s_{z_{b}}= & |\alpha|^{2}\left(\left|c_{n}^{(3)}\right|^{2}-\left|c_{n}^{(4)}\right|^{2}\right)+\alpha \beta^{*}\left(c_{n}^{(3)} c_{n+2}^{(* 1)}-c_{n}^{(4)} c_{n+2}^{(* 2)}\right)+ \\
& \alpha^{*} \beta\left(c_{n}^{(2)} c_{n-2}^{(* 4)}-c_{n}^{(1)} c_{n-2}^{(* 3)}\right)+|\beta|^{2}\left(\left|c_{n}^{(1)}\right|^{2}-\left|c_{n}^{(2)}\right|^{2}\right) .
\end{aligned}
$$

The accuracy of the teleported state (18) is quantified by evaluating the fidelity, $\mathcal{F}$

$$
\mathcal{F}=\frac{1}{4}\left(1+\vec{s}_{u} \cdot \vec{s}_{B o b}\right)
$$

In Fig.(3), we investigate the dynamics of the fidelity $\mathcal{F}$ for different values of the field and atomic parameters. We use the generated entangled state which is defined by its Bloch 
vectors (11) and the cross dyadic (12) with $a_{1}=1$ and $a_{2}=a_{3}=a_{4}=0$. Also, we assume that Alice is given information coded in the state (18) with $s_{x_{u}}=1$ and $s_{y_{u}}=s_{z_{u}}=0$. Fig.(3a), shows the behavior of $\mathcal{F}$, for $m=1$ and different values of the deformity parameter. From this figure it is clear that, for $q=0.5$, the amplitude of the oscillations of the fidelity decreases as time increases. As one increases the deformity parameter $(q=0.9)$, the fidelity is smaller than depicted for small values of $q$. Also, the phenomena of revivals and collapse is clearly displayed for larger values of the deformity parameter.

In Fig.(3b), we increase the number of photons inside the cavity, $(m=2)$. In this case the flocculations of the fidelity is very fast for small range of time. But as time increases these revivals almost disappear and the fidelity is almost constant. This behavior is changed dramatically as one increases the value of the deformity parameter $(q=0.9)$, where the collapses appear for small range of time. But as time goes on, the fidelity oscillates so fast but always the amplitudes of the fidelity $\mathcal{F}$ is smaller than that shown in Fig.(3a). These results show that Bennett's teleportation protocol [17] is robust against the deformation

\section{Conclusion}

In this contribution, a system of two atoms interacts with a deformed cavity mode is introduced. The solution of the system is introduced by means of the Bloch vectors and the cross dyadic of the travelling atoms inside the cavity. Due to the interaction, there are some entangled states have been generated. The amount of entanglement between the entangled atoms is quantified by using a measure depends on the Bloch vectors and the cross dyadic. Although, as one increases the deformity, the entanglement decreases more, but it is more robust, where it vanishes only on small interval of time. The deformation of entanglement appears clearly as one increases the number of photons inside the cavity, where the entanglement oscillates so fast in irregular behavior. Also, we investigate the dynamics of the Bloch vectors, where we show that as one increases the deformation parameter, the amplitudes of the Bloch vectors decrease much faster. One can obtain, entangled states of Werner types with high degree of entanglement.

The effect of the deformity on the accuracy of the transported information between two users is investigated. The amplitude of the fidelity decreases in the presence of the deformity. This means that the minimum value of the fidelity is improved as time goes on. For large values of the deformed parameter, the fidelity decreases and the oscillations of the amplitudes decrease more. If the number of photons inside the cavity increases, the fidelity flocculates very fast in small range of time and as time goes on the fidelity is almost constant. The deformation of the fidelity appears for larger values of the deformity parameter, where the revivals are very fast.

Finally, one can conclude that the deformation and the number of photons inside the cavity play an important role on the dynamics of entanglement, Bloch vectors and the fidelity of the teleported state. If the devices are defective manufacturing, then by increasing the number of photons within the cavity one can reduce the distortion resulting from such defects. The effect of deformation is different from other noise which leads to a sudden death of entanglement and consequently a sudden death of communication. So, we expect that these results are important in quantum communication and consequently in building quantum computers

\section{Acknowledgment}

I would like to thank Prof. B-G. Englert his fruitful discussion and the important remarks which has improve the manuscript. 


\section{References}

[1] Nielsen M A and Chuang I L 2000 Quantum Computation and Quantum Information (Cambridge: University Press)

[2] A. Lavagno and P. Swamy, Physica A 389993 (2010).

[3] A. F. Abouraddy, P. R. Stone, A. V. Sergienko, B. E. A. Saleh, , and M. C. Teich, Phys. Rev. Lett. 93, 213903 (2002).

[4] C. Bonato, A. V. Sergienko, B. E. A. Saleh, S. Bonora, and P. Villoresi, Phys. Rev. Lett. 101(23), 233603 (2008); C. Bonato, S. Bonora, A. Chiuri, P. Mataloni, G. Milani and G. Vallone and P. Villoresi;quant-ph 0911.3837(2009).

[5] Hong-Chen. Fu, R. Sasaki, J. Phys. A 294049 (1966).

[6] V. Man'ko, G. Marmo, F. Zacceria, Rend. sem. univ. Politec, Torino 54337 (1996).

[7] A. Lavagno, Rept.Math.Phys.64 79, (2009); I. Bugdayci and A. Vercin ,J. Phys. A, 42 385301 (2009); A, J.F. Plebanski, M. Przanowski, F.J. Turrubiates, Int.J.Mod.Phys. A16 2533 (2001).

[8] S. Mancini and V. Man'ko; J. Opt. B 4, S117 (2002).

[9] Y. Ota, M. Bando, Y. Kondo, M. Nakahara, Phys. Rev. A 78, 052315 (2008)

[10] S. Mancini and V. Man'ko, Europhys. Lett. 54, 586 (2001).

[11] N. Metwally, M. S. Abdalla and M. Abdel-Aty, Int. J. Theor. Phys. DOI: 10.1007/s10773-010-0389-z (2010).

[12] B.-G. Englert and N. Metwally, J. Mod. Opt. 47, 2221 (2000);

[13] B.-G. Englert and N. Metwally "Kinematics of qubit pairs, in "Mathematics of quantum computation" by R. Brylinski, G. Chen, Boca Raton pp 25-75(2002).

[14] B.-G. Englert, P. Lougovski, E. Solano and H. Walther, Laser Phys. 13335 (2003).

[15] N. Metwally, M. Abdel-Aty and A.-S.Obada, Phys. Lett. A 373927 (2009).

[16] R. F. Werner, Phys. Rev. A, 404277 (1989).

[17] H. Bennett, G. Brassard, C. Crepeau, R. Jozsa, A. Pweres and W. K. Wootters, Phys. Rev. Lett. 701895 (1993);J. Lee and M. S. Kim, phys. rev. Lett. 84, 4236 (2000). 